\title{
La deliberación pública en el proceso colectivo de recomposición ambiental
}

Public deliberation in the collective process of environmental recomposition

Deliberação pública no processo coletivo de recomposição ambiental

Délibération publique dans le processus collectif de recomposition environnementale

\section{Ezequiel R. Galván ${ }^{1}$ | Universidad Nacional de La Plata}

Revista Derechos en Acción / ISSN 2525-1678 / e-ISSN 2525-1686

Año 4/NNo 10 Verano 2018/2019 (21 diciembre a 21 marzo), 217-233

DOl: https://doi.org/10.24215/25251678e251

ORCID: https://orcid.org/0000-0003-0833-4366

Recibido: 18/12/2018

Aprobado: 03/03/2019

Resumen: En el presente trabajo se aborda las particularidades del proceso de recomposición ambiental entendiendo al mismo como un proceso necesariamente colectivo en el cual debe existir una instancia de deliberación pública previa a la sentencia que ordene medidas de recomposición. Asimismo se intenta recuperar la experiencia de la causa "Mendoza" como modelo de referencia tanto por el proceso que sustanciaba como por la estructura de proceso que se implementó. En este sentido, ante la ausencia de una reglamentación adecuada, la construcción de un modelo a partir de una experiencia precedente y legitimada por la comunidad en su conjunto (sin desconocer las limitaciones o falta de efectividad en el cumplimiento de su mandato) permite construir y sustanciar procesos con mayores posibilidades de efectividad ante obstáculos o limitaciones que atentan contra la implementación de un proceso de esta naturaleza y no condicionándolo a la casuística del caso.

1 Abogado (UNLP). Escribano (UNLP). Diplomado en DESC (UM). Maestrando en Derechos Humanos (IDH-UNLP). mail: ezequielrgalvan@gmail.com . 
Palabras claves: Deliberación pública. Medio ambiente. Proceso colectivo.

Abstract: In the present work, we will focus in the particularities of the environmental recomposition process, understanding it as a necessary collective process in which there must be an instance of public deliberation prior to the ruling that orders reconstruction measures. At the same time it seeks to incorporate the experience of the cause "Mendoza" as a model of reference both for the process that substantiated and for the process structure that was implemented. In this sense, in the absence of an appropriate regulation, the construction of a model based on a previous experience and legitimized by the community as a whole (without ignoring the limitations in fulfilling its mandate) allows to build and substantiate processes with greater possibilities of impact before obstacles or limitations that threaten the implementation of a process of this nature and not conditioning it to the the casuistry of the case.

Keywords: Public deliberation. Environment. Collective process

Resumo: Neste trabalho é analizado as particularidades do processo de recomposición ambientais, entendê-lo como um processo necessariamente coletivo en que deve ser uma instância de deliberação pública antes da sentença que ordene medidas de reestruturação. Da mesma forma, tenta-se recuperar a experiência da causa "Mendoza" como modelo de referência tanto para o processo que fundamentou, como para a estrutura de processo que foi implementada. Nesse sentido, na ausência de regulação adequada, a construção de um modelo baseado em uma experiência única e legitimada pela comunidade como um todo (sem desconsiderar as limitações de efetividade no cumprimento de seu mandato) permite a construção e subestação de processos com maior possibilidades de eficácia perante obstáculos ou limitações que atentam contra a implementação de um processo desta natureza e não o condicionam à casuística do caso.

Palavras-chave: Deliberação pública. Meio Ambiente. Processo coletivo

Résumé: Dans cet article, nous analysons les particularités du processus de recomposition environnementale, comme un processus nécessairement collectif dans lequel il doit y avoir une instance de délibération publique avant la condamnation ordonnant des mesures de recomposition. Dans le même temps, nous essayons de récupérer l'expérience de 
la cause "Mendoza" en tant que modèle de référence à la fois pour le processus qu'elle justifie et pour la structure de processus mise en place. En l'absence d'une réglementation adéquate, la construction d'un modèle basé sur une expérience antérieure et légitimée par la communauté dans son ensemble (sans ignorer les limitations ou le manque d'efficacité dans la réalisation de son mandat) permet de construire et de justifier des processus avec une possibilités d'efficacité avant les obstacles ou limitations qui s'opposent à la mise en œuvre d'un processus de cette nature et ne le subordonnent pas à la casuistique du cas.

Mot-clés: Délibération publique. Environnement. Processus collectif

\section{Introducción}

El presente trabajo aborda las particularidades del proceso de recomposición ambiental entendiendo al mismo como un proceso necesariamente colectivo en el cual debe existir una instancia de deliberación pública previa a la sentencia que ordene medidas de recomposición. En este sentido, a partir de la última reforma constitucional se introdujo una concepción del ambiente entendiéndolo como un bien colectivo ${ }^{2}$ que se presenta paradigmático debido a que su gestión presenta un carácter deliberativo público y directo que en el presente denominamos "participación ciudadana" o "deliberación pública" (indistintamente) ${ }^{3}$, rompiendo con el precepto constitucional por el cual "el pueblo no delibera ni gobierna, sino por medio de sus representantes y autoridades creadas por esta Constitución"

2 Como expresa la Corte Suprema de Justicia de la Nación: "La mejora o degradación del ambiente beneficia o perjudica a toda la población, porque es un bien que pertenece a la esfera social y transindividual, y de allí deriva la particular energía con que los jueces deben actuar para hacer efectivos estos mandatos constitucionales." (CSJN, "“Mendoza, Beatriz Silvia y otros c/ Estado Nacional y otros s/daños y perjuicios (daños derivados de la contaminación ambiental del Río Matanza - Riachuelo)" sentencia de 20-VI-2006, considerando 18.

3 Se evita el término "audiencia pública" debido a que se entiende a esta como una modalidad de la participación ciudadana, existiendo otros mecanismos para su sustanciación, ej. la recepción de observaciones escritas (arts. 44 y 73 inc. 2 Reglamento de la Corte Interamericana 2009). 
(art. 22 C.N.) y modifica la estructura del proceso como consecuencia del mandato constitucional vigente.

La principal consecuencia de entender al ambiente como un bien colectivo es que el proceso que aborde la recomposición del daño ambiental necesariamente va a ser un proceso colectivo (criterio que recoge el art. 30 de la ley 25.675), teniendo presente que: a) la pretensión aborda derechos de incidencia colectiva que tienen por objeto bienes colectivos, es decir, la petición tiene por objeto la tutela de un bien que pertenece a toda la comunidad, indivisible y que no admite exclusión alguna; b) la pretensión se focaliza en la incidencia colectiva del derecho (recomposición) y no en dimensiones individuales pasibles de un abordaje individual independiente (ej. indemnización de daños producto del impacto ambiental); c) el ejercicio de la pretensión procesal de reparación del bien colectivo obtiene una decisión cuyos efectos repercuten sobre el objeto de tutela sin generar un beneficio directo para el individuo que ostenta la legitimación (accionante) ${ }^{4}$.

Se tiene presente que los procesos colectivos representan un carácter atípico dentro de la lógica clásica, caracterizados por una ausencia de regulación, salvo algunas disposiciones específicas, por ejemplo, en la ley general del ambiente (Verbic, 2015) y una instrumentación ad-hoc por la jurisdicción ${ }^{5}$. En este sentido, el presente trabajo no pretende controvertir la construcción teórica y jurisprudencial en torno al proceso colectivo y la ficción que lo sustenta de un actor que se constituye en representante de un colectivo sin la integración de los mismos al proceso (como se expondrá más avanzado este texto), sino que se intenta una construcción teórica que

4 CSJN "Halabi, Ernesto c/ P.E.N. - ley 25.873 - dto. 1563/04 s/ amparo ley 16.986", sentencia de 24-II-2009, considerando 11.

5 "Desde la perspectiva remedial, la nueva modalidad de litigio no supone la compensación de daños pasados y acotables a las partes, sino la transformación hacia el futuro de prácticas institucionales a través del diseño ad hoc de soluciones cuyas consecuencias excederán, en la mayoría de las situaciones, el impacto en las partes presentes ante el juez" (Bergallo, 2015:18) 
resulte coherente y armónica con el proceso colectivo vigente y su práctica, teniendo como pauta rectora procurar "la vigencia del principio de economía procesal, a la efectividad del acceso a la jurisdicción (arts. 18, Const. Nac.; 8 y 25, Conv. Americana sobre Derechos Humanos; 15, Const. de la Provincia de Buenos Aires), y a la disuasión de infracciones que de otro modo permanecerian impunes" (Giannini, 2009).

Finalmente, antes de ingresar en el objeto específico del trabajo, el mismo se restringió por cuestiones metodológicas a los procesos de recomposición ambiental sin abordar aquellos que se restringen al cese de una actividad lesiva sin una instancia de recomposición, las acciones que reclaman daños ambientales colectivos en un sentido indemnizatorio (entendiendo la diferencia con la recomposición en tanto que la misma tiene una proyección del bien hacia el futuro, mientras que el daño colectivo reclamado se presenta como una pretensión de derechos individuales homogéneos condicionados por la constatación del hecho productor, nexo causal y entidad del daño sufrido respecto de cada individuo $)^{6}$, así como las acciones abstractas de inconstitucionalidad ${ }^{7}$ respecto de la legislación ambiental (leyes o reglamentaciones) debido a que independientemente de que podamos estar ante un proceso colectivo ambiental, estas acciones no requieren una deliberación pública necesaria por un imperativo constitucional que sí se encuentra presente en las acciones de recomposición, como se desarrolla a continuación.

\section{El proceso colectivo de recomposición ambiental y la deliberación pública}

Ingresando en el objeto específico del trabajo y recuperando el desarrollo introductorio, la última reforma constitucional

\footnotetext{
6 CSJN “Mendoza” 2006 cit., considerando 6 y 8.

7 Abstracta en un sentido fáctico dado por la ausencia de una situación de impacto ambiental concreta (ante el daño o en una faz preventiva) y no en un sentido técnico de "ausencia de caso" en la acción que excluiría su tratamiento por la jurisdicción.
} 
exhibe una resignificación constituyente del concepto de democracia, entendiéndolo no solo en una dimensión formal dada por la continuidad de la institucionalidad representativa democrática y constitucionalmente electa, sino también en un sentido material dado por la apertura de distintos espacios de gobierno a la participación activa de la ciudadanía ${ }^{8}$. Es así como encontramos en el capítulo "Nuevos Derechos y Garantías" un derecho de la ciudadanía a resistir la interrupción del orden institucional y democrático (art.36), se reafirma el principio de soberanía popular ahora entendiendo la participación en la vida política/ pública en un sentido material -remoción de obstáculos en su ejercicio pleno- (art.37), el derecho de asociarse para participar en el sistema democrático en igualdad de condiciones (art.38), la iniciativa popular en materia legislativa (art.39), la consulta popular (art.40), el derecho colectivo al ambiente (art.41), los derechos de los consumidores con instancias de participación (art.42), y el derecho a un debido proceso colectivo (art.43) (Verbic, 2015).

En el caso particular del ambiente, el reconocimiento fue acompañado de la introducción de un mecanismo de protección denominado "presupuestos mínimos" por el cual el Congreso de la Nación establece los estándares mínimos inderogables e indisponibles por las provincias ${ }^{9}$ a través de leyes, entre las que se destaca el dictado de la 25.675, una ley de carácter general y preeminente (art.3) que consagra expresamente el rol central de la participación ciudadana (arts.19-21) como principio rector en la gestión ambiental.

No se desconoce que la ley 25.675 hace referencia expresamente a la deliberación pública en el marco de procedimientos administrativos, sin embargo, se sostiene su extensión a los

8 El art. 42 ( $3^{\circ}$ párrafo) de la Constitución es un claro ejemplo al establecer la participación de las asociaciones de consumidores y usuarios en los órganos de control de los servicios públicos de competencia nacional.

9 CSJN, A.262.XLV “Asociación Argentina de Abogados Ambientalistas c/ Buenos Aires, Prov. y otros s/ amparo ambiental", sentencia de 01-XI2011, considerando 6. 
procesos judiciales de recomposición ambiental debido a que esta participación se exige obligatoriamente en los procesos de decisión sobre actos concretos por los que se preserva, protege (art. 19), se autorizan actividades que pueden generar un impacto negativo y significativo (art. 20), así como en las evaluaciones de impacto ambiental (supervisión previa o de seguimiento de la actividad pasible a generar ese impacto negativo), y programas o planes de ordenamiento ambiental territorial, tanto en su planeamiento como en su evaluación (art. 21), con una clara restricción del poder de gobierno de la administración pública (representantes) en materia de gestión del ambiente. En este aspecto, se entiende por "gestión" como la decisión sobre el destino del ambiente y las conductas que pueden tener impacto en el mismo, la cual radica en la administración pública como consecuencia de la división de poderes, pero que se integra esa voluntad con esta instancia de apertura a la participación directa de la sociedad por mandato legislativo.

En la interpretación que el presente trabajo sostiene, esta participación pública establecida no se origina en una liberalidad legislativa, sino en el propio carácter colectivo del bien, el cual no es pasible de apropiación individual (incluyendo su gestión) por ningún sujeto, incluyendo al Estado y las Provincias ${ }^{10}$. En este sentido, es importante tener presente que el derecho ambiental presenta una naturaleza dialéctica (Bibiloni, 2005) dada por la existencia de una pluralidad de intereses contrapuestos. En consonancia con lo expuesto, la instancia judicial es, en definitiva, el abordaje de una afectación al ambiente a través de un impacto cuya gestión ordinaria establece una necesaria autorización administrativa con una participación ciudadana

\footnotetext{
10 También podemos encontrar otras posiciones que fundamentan esta participación en el derecho a participar de los asuntos públicos, por ejemplo, "del derecho de participación en los asuntos públicos, deriva la obligación de los Estados de garantizar la participación de las personas bajo su jurisdicción en la toma de decisiones y políticas que pueden afectar el medio ambiente, sin discriminación, de manera equitativa, significativa y transparente, para lo cual previamente deben haber garantizado el acceso a la información relevante" (ColDH, 0.C.23/17, párrafo 231).
} 
directa (deliberativa), por lo que no puede despojarse de esta instancia el proceso judicial en tanto que suple la omisión o insuficiencia del control ordinario administrativo, con una sentencia que dispone la gestión final del ambiente en el caso concreto.

A diferencia de otras instancias que requieren una deliberación pública obligatoria (ej. aumento de $\operatorname{tarifas}^{11} \mathrm{o}$ permisos ambientales $^{12}$ ) en las que la Corte Suprema ha nulificado lo actuado y mandado a sustanciar dicha participación en el marco de una instancia administrativa, el proceso de recomposición debe sustanciar dicha participación en el marco del proceso como consecuencia de una gestión del ambiente que va a ser ordenada por el juez, en contraposición a los otros casos en los que la decisión en la materia radica en la administración pública sin que el poder judicial reemplace dicha función y restringiendo su accionar a un contralor de la legitimidad del acto (Gordillo, 2013). ${ }^{13}$

Debe tenerse presente que participación ciudadana no debe confundirse con la legitimación o carácter de parte en el proceso. El proceso colectivo se estructura alrededor de la ficción por la cual un representante actúa a nombre de un colectivo, incluso con el desconocimiento de las personas que lo integran, en el marco de un proceso con sentencia cuyos efectos alcanzan al grupo representado. En este sentido, a quien ejerza esa legitimación colectiva se le requerirán una serie de requisitos (legitimación, representatividad adecuada, publicidad, cuestión de hecho o derecho principal de origen común, identificación del grupo, posibilidad de ejercer el "opt out" en los casos que sean posibles -pues el ambiente en su faz de recomposición no permite un accionar diferenciado por quien intente excluirse-, e imposibilidad o grave dificultad para constituir un

\footnotetext{
11 CSJN, “Centro de Estudios para la Promoción de la Igualdad y la Solidaridad y otros e/ Ministerio de Energia y Mineria s/ amparo colectivo", sentencia de 18-VIII-2016.

12 CSJN, "Asociación argentina de abogados ambientalistas de la Patagonia c/ Santa Cruz, Provincia de y otro s/ amparo ambiental", sentencia de 21-XII-2016.

13 Mientras estos actos producen sus efectos a futuro, el proceso de recomposición aborda un daño existente.
} 
litisconsorcio o un ejercicio individual de los derechos que constituyen la cuestión predominante de la pretensión) va a ser "parte" del proceso en términos procesales, con capacidad de peticionar e intervenir en el proceso.

En el caso de la participación pública, la misma constituye una instancia procesal en la que se produce una apertura del juzgado a la recepción de las consideraciones de la sociedad en general respecto de la materia sometida a dicha participación (las medidas de recomposición y/o el plan de trabajo propuesto) para que formulen sus apreciaciones ${ }^{14}$, sin que esto implique reconocerles calidad de parte o mayor facultad para intervenir en el proceso que un amicus curiae (recuperando el instituto como una figura de naturaleza asimilable en cuanto es una participación restringida a incorporar voces sin reconocerle facultad de intervención en el desarrollo del proceso). Si bien se expondrán las particularidades y propuesta de abordaje de esta instancia en mayor detalle, como puede observarse no se adultera con el reconocimiento de esta instancia los caracteres propios del proceso colectivo

\section{La instancia de deliberación pública dentro del proceso: propuesta y sus fundamentos}

Partiendo de la necesidad de sustanciar la participación ciudadana en el marco de un proceso de recomposición ambiental, la propuesta del presente trabajo para su instrumentación en el marco del proceso busca una coherencia con la regulación vigente y los principios que rigen el proceso colectivo. De este

\footnotetext{
14 Aún con un alcance restringido a los afectados directos en contracara al propuesto en el presente trabajo, podemos encontrar la implementación de una instancia de participación ciudadana en los programas de recomposición, así como el reconocimiento de una pluralidad de sujetos con posiciones contradictorias cuya voz se reconoce como relevante en la implementación de una sentencia sin que implique desdibujar los límites del proceso colectivo y la representación de esa pluralidad en el sujeto representante "parte" a los efectos procesales: "deberá supervisarse el traslado de los vecinos de Villa Inflamable, resguardando su derecho participar en las decisiones que las autoridades encargadas adopten en el curso del proceso de reubicación." CSJN, "Mendoza" sentencia de 19-XII-2012, considerando 6 p.b).
} 
modo, la instancia de deliberación pública (en nuestro criterio) correspondería sustanciarla de modo posterior a una primer sentencia de contenido restringido a identificar la procedencia de la pretensión principal (mandato de cese y recomposición) que difiere la instrumentación de las medidas específicas ha implementarse $^{15}$. En este aspecto, la propuesta de un proceso de etapas (sentencias) responde al desarrollo de un litigio que aborda situaciones estructurales y/o complejas que exceden la estructura del litigio individual, siendo la causa "MendozaRiachuelo" ante la Corte Suprema un precedente paradigmático tanto de la implementación de un proceso ad-hoc a los fines de tutelar ante la ausencia de proceso efectivo ${ }^{16}$, como de un proceso complejo de etapas.

El fundamento por el que se propone la sustanciación de esta instancia de modo posterior a una primera sentencia está dado por un principio de celeridad y economía procesal, así como por no desnaturalizar la estructura del proceso colectivo ambiental. De este modo, la primera etapa del proceso se integra con un sujeto legitimado con representatividad adecuada que impulsa una acción colectiva para obtener el cese de la actividad dañosa (si se continua) y la recomposición del ambiente, culminando con una sentencia que va a pronunciarse sobre la existencia de un daño y la responsabilidad de la parte demandada. En este sentido, la sentencia puede entender tanto la inexistencia del daño como la ausencia de responsabilidad o legitimación pasiva de la contraparte, generando un efecto de cosa juzgada propia del proceso ambiental que va a poder ser reeditada solo respecto de su rechazo total o parcial por cuestiones probatorias (art. 33 ley 25.675), así como entender

\footnotetext{
15 "Una de dichas herramientas de "saneamiento" es la posibilidad de escisión del pronunciamiento condenatorio grupal en fases. Esta separación tiene por objeto el avance progresivo del juicio sobre etapas sólidamente asentadas, cerrando el debate acerca de la determinación del an debeatur a partir del dictado de un primer decisorio (condena genérica), para después poder ingresar en los matices de cada situación particular." (Giannini, 2009: 23). 16 Entendiéndolo en un sentido convencional (ColDH, 0.C. 09/87, párrafo 23).
} 
probado el daño y conductas lesivas, circunstancia en la que estaremos ante una sentencia atípica y declarativa con las bases de su ejecución (Cafferatta, 2008).

Como podemos observar, siendo que la participación ciudadana se requiere en la decisión sobre gestión del ambiente y no en el proceso colectivo en sí, donde "el derecho a ser oído por el juez se limita aquí a ser oído a través de un atípico gestor de intereses ajenos" (Verbic, 2015:14), su sustanciación en esta instancia se presenta contraproducente debido a que dilata una sentencia que hace mérito de esa pretensión principal de recomponer sin contribuir a la misma ${ }^{17}$ y generando un dispendio de la jurisdicción ante el dictado de una sentencia que rechace la acción. De igual modo, el dictado de esta primera sentencia permite tanto avanzar sobre las medidas inmediatas respecto del cese de la actividad dañosa y medidas que eviten una mayor afectación (sin avanzar en la tutela cautelar durante el proceso que excede al presente trabajo y no se exhibe pertinente dados los objetivos del mismo).

Dictada la sentencia de condena a recomponer ${ }^{18}$, se abre una instancia atípica de ejecución de sentencia cuyo objetivo es instrumentar las medidas específicas de recomposición. Es en esta instancia que nosotros entendemos necesaria (en el sentido de su sustanciación obligatoria) la participación ciudadana, la cual puede variar en los medios de su implementación ${ }^{19}$, pero

17 Es importante tener bien definido que la participación ciudadana no constituye un litisconsorcio que integra el proceso junto al legitimado con representatividad adecuada, quien es el único que puede peticionar, alegrar, sustanciar prueba e impulsar el proceso, sin perjuicio de aquellos que deseen intervenir en el proceso y se presenten en el mismo en el carácter que invoquen (se consideren terceros, deseen reemplazar al actor por considerarse con mejor representación, etc.), es decir, no se modifica la lógica constitutiva del proceso colectivo.

18 "Emite una orden, un mandato de condena, como sentencia definitiva: recomponer y prevenir. Y mejorar a calidad de vida de la población. Pero no invade ámbitos de poder que no le son propios. Deja en manos del Ejecutivo el desarrollo modal de la obra." (Cafferata, 2008: 147).

19 La participación ciudadana tiene un carácter sustantivo cuyo contenido es independiente de su continente, pudiendo sustanciarse por audiencias públicas, observaciones escritas, 
tiene requisitos necesarios que serán transversales al desarrollo de esta etapa. El primer requisito es la publicidad de esta instancia, tanto respecto de la apertura de esta instancia y sus modalidades para participar, así como del contenido y alcance de la sentencia (mandato a ejecutarse) ${ }^{20}$. El segundo requisito es que es la posibilidad efectiva de participación y el tercer requisito es que estas expresiones sean consideradas en el momento decisorio ${ }^{21}$. Por lo tanto, de los requisitos sustanciales de la participación ciudadana deriva una estructura de esta instancia procesal cuyas etapas son: a) implementación de la participación ciudadana (plazo, modalidad y otros aspectos prácticos), también pudiendo estar definidos en la sentencia declarativa previa; b) publicidad de la instancia de deliberación así como de la información pertinente; c) sustanciación de la

organismos o asistentes técnicos a los que el juez le delegue la recopilación de los planteos ciudadanos, entre otras.

20 La importancia de la publicidad de la instancia (apertura, modalidad, límites de la discusión) tiene una dimensión que está dada por las posibilidades efectivas del ejercicio del derecho que su sustanciación representa en un sentido material. Esta apertura debe instrumentarse de un modo por el que se remuevan los obstáculos para ejercer este derecho, especialmente respecto de las personas con un contacto más directo con el ámbito de las medidas (recuperando en este aspecto también los debates existentes respecto de la publicidad de la promoción del proceso colectivo). Una instrumentación que no sea contemplativa de esta dimensión puede derivar en una práctica discriminatoria, por ejemplo, ¿el mero dictado de la sentencia o su publicación en el Boletín Oficial es una publicidad suficiente? $\mathrm{Si}$ se disponen medidas que repercuten en mayor medida sobre el espacio en el que se asienta un colectivo marginal alejado del juzgado existen limitaciones fácticas tales como el desconocimiento de la existencia del proceso, la sentencia o la convocatoria, personas analfabetas que se vean excluidas por una publicidad o una recepción escrita de sus voces, las dificultades de traslado al juzgado, entre otras que demuestran la necesidad de una instrumentación situada con esta perspectiva.

21 CSJN, "Centro de Estudios para la Promoción de la Igualdad y la Solidaridad" cit., considerando 19. Si bien el fallo aborda la audiencia pública en el marco del cuadro tarifario (art. 42 C.N.) la Corte establece requisitos sustanciales que estas instancias deliberativas "si lo que genuinamente se persigue es profundizar el fiel ejercicio de derechos por parte de los ciudadanos en una sociedad democrática, y no acrecentar por parte de los poderes políticos su catálogo formal de instituciones nominales vaciadas de todo contenido, que únicamente aumentan sus credenciales democráticas y que solo pretenden legitimar decisiones verticales tomadas con anterioridad." 
participación ciudadana; d) abordaje de los planteos y sentencia con las medidas específicas de recomposición a implementarse.

La recomposición ambiental es un proceso complejo que requiere el diseño de un programa de trabajo, lo que motiva que el juez no determine el programa a implementarse, sino que analice la pertinencia de un proyecto producido por un cuerpo técnico $^{22}$. En este sentido, nuestra propuesta sitúa este desarrollo del programa específico como simbiótico a la participación ciudadana en tanto que va a generar la información y medidas principales propuestas que van a representar esa información previa y plena que requiere la ciudadanía para su efectiva participación (primer requisito), así como va a ser la instancia que recepcione esas intervenciones en la construcción del programa final, siendo el juez un director del proceso así como contralor tanto de la cumplimiento del mandato genérico de esa primer sentencia por el programa de recomposición propuesto y de un efectivo abordaje de la intervención de la ciudadanía ${ }^{23}$.

Si bien la sustanciación de la participación ciudadana en esta instancia judicial la entendemos necesaria, esto no significa que la autoridad judicial no pueda delegar en otro organismo el desarrollo de esta instancia ${ }^{24}$, lo que representa una delegación práctica debido a que el poder de decisión se conserva

\footnotetext{
22 Además del marco teórico a partir del cual se construye la propuesta del presente trabajo se recupera la causa "Mendoza" como modelo de referencia de un proceso colectivo de recomposición ambiental, debido a que constituye una práctica que expone el carácter complejo y de etapas en la implementación de las medidas que requiere la ejecución de la sentencia.

23 El abordaje de estas intervenciones no es entenderlas en un sentido vinculante, sino en que haya una respuesta fundada de aquellos aspectos que no se consideren pertinentes a la situación, recupere las preocupaciones sociales expresadas y dialogue con esa ciudadanía, generando un producto que no sea arbitrario o constituya la instancia en un mero ritualismo.

24 "A los fines de garantizar la efectividad en el cumplimiento de la sentencia, esta Corte (...) habilitó la participación ciudadana en el control del cumplimiento con la intervención del Defensor del Pueblo de la Nación de las organizaciones no gubernamentales interesadas en la tutela del ambiente" (CSJN “Mendoza" sentencia de 19-XII-2012, considerando 1).
} 
en la autoridad judicial ${ }^{25}$. Es importante tener presente que los procesos colectivos complejos (en cuanto a la entidad de la pretensión) o de litigio estructural requieren un abordaje en miras a su efecto útil, especialmente porque la propia naturaleza del mandato judicial restringe su cumplimiento coactivo a instancias del poder judicial ${ }^{26}$.

Si bien el presente trabajo tiene un postulado transversal dado por entender la participación ciudadana como necesaria en estos procesos, la necesidad de generar mecanismos y modificaciones en las estructuras procesales que permitan responder ante una vulneración de derechos en ausencia de estructuras adecuadas ha sido una constante en la evolución de nuestro ordenamiento jurídico. De este modo, encontramos dentro de la práctica judicial sentencias paradigmáticas que readecuan un continente en pos de un contenido, en tanto que "Los preceptos constitucionales tanto como la experiencia institucional del país reclaman de consuno el goce y ejercicio pleno de las garantías individuales para la efectiva vigencia del Estado de derecho e imponen a los jueces el deber de asegurarlas." ${ }^{27}$

\section{Conclusiones}

En el presente trabajo hemos intentado demostrar la necesidad de sustanciar la participación ciudadana en el marco de los procesos de recomposición ambiental (proceso que por naturaleza es colectivo) como cumplimiento de un mandato

\footnotetext{
25 "la formulación de la sentencia en el litigio de interés público introduce una gran cuota de control de las partes sobre el resultado práctico del proceso (...) Sin embargo, no puede suponerse que este proceso relevará al tribunal por completo de la responsabilidad de diseñar la remediación." (Chayes, 2017:20-21).

26 "No puede pretenderse que un proceso ideado para discutir derechos individuales pueda brindar una respuesta adecuada a la hora de resolver asuntos que involucran derechos "de incidencia colectiva" Las complejas aristas sociales, políticas y económicas que presentan los procesos colectivos (ambientales y no ambientales) exigen un mecanismo de discusión rodeado de mayor transparencia, publicidad y ciertos reaseguros que permitan solucionar el conflicto sin vulnerar la garantía de debido proceso legal de las personas involucradas." (Verbic, 2013:282). 27 "Siri, Juan Angel s/ interpone recurso de habeas corpus" sentencia de 27-XII-1957.
} 
constitucional y legislativo. En este sentido, la última reforma constitucional incorporó una concepción de democracia que reconoce la apertura de espacios de gobierno y decisión a la participación política activa e incluso directa de la sociedad, lo que obliga a adecuar las estructuras e instituciones a esta apertura.

El proceso colectivo constituye una garantía constitucional de protección y tutela, cuya implementación no puede ser restringida por la ausencia de normas procesales adecuadas. La propia naturaleza del proceso colectivo y del litigio estructural demanda que la autoridad judicial arbitre los medios necesarios para sustanciar esa garantía reconocida, con un rol y estructuras que exceden la lógica clásica del proceso individual. En este sentido, la instrumentación de la participación ciudadana se inscribe en este mandato a la judicatura.

Cómo se ha intentado demostrar, la participación ciudadana en la gestión del ambiente en el marco de un proceso colectivo no modifica la lógica del proceso colectivo o la legitimación de un sujeto que actúa a nombre de un grupo o colectivo como representante. La intervención en el proceso se encuentra restringida al representante, así como a los demás actores que se integren al mismo con la legitimación y calidad que invoquen (cuya resolución es ajena e intrascendente a la instancia de deliberación pública).

La participación de la ciudadanía es una instancia que se encuentra restringida al diseño de las medidas de gestión del ambiente, las cuales tienen requisitos sustantivos que repercuten en la estructura del proceso. En esta línea, la instrumentación del proceso debe garantizar la posibilidad efectiva de participación (especialmente de quienes tienen una relación más directa con la problemática) pero con una estructura que responda a los principios que rigen el proceso colectivo. La apertura de la decisión además de tener un valor en términos democráticos, también contribuye a reconstruir la complejidad de una situación con pluralidad de actores, intereses e inquietudes/ aspiraciones, enriqueciendo la calidad de la respuesta judicial. 
Si bien el proceso colectivo se sustancia bajo un representante que enarbola una pretensión colectiva común (recomponer el ambiente) es importante reflexionar la representatividad de ese colectivo de posiciones contradictorias sobre la gestión del ambiente (medidas específicas) de quien impulsa el proceso. Esto último no significa desconocer la legitimación de quien impulsa el proceso ambiental, sino entender que la propia naturaleza del conflicto genera ámbitos que exceden la litis trabada entre quien acciona y quien es requerido, carácter que es reconocido en la ley 25.675: "Asimismo, en su Sentencia, de acuerdo a las reglas de la sana crítica, el juez podrá extender su fallo a cuestiones no sometidas expresamente su consideración por las partes" (art. 32).

Finalmente, el presente trabajo recupera la experiencia de la causa "Mendoza" como modelo de referencia tanto por el proceso que sustanciaba como por la estructura de proceso que se implementó. En este sentido, ante la ausencia de una reglamentación adecuada, la construcción de un modelo a partir de una experiencia precedente y legitimada por la comunidad en su conjunto (sin desconocer las limitaciones o falta de efectividad en el cumplimiento de su mandato) permite construir y sustanciar procesos con mayores posibilidades de efectividad ante obstáculos o limitaciones que atentan contra la implementación de un proceso de esta naturaleza y no condicionándolo a la casuística del caso ${ }^{28}$.

\section{Bibliografía}

Bergallo, P. (2005) “Justicia y experimentalismo: la función remedial del poder judicial en el litigio de derecho público en Argentina"

\footnotetext{
28 "una nota de advertencia que creo necesaria: la CSJN puede gestionar el proceso como lo está haciendo porque es la CSJN. Lo que quiero decir es que difícilmente encontremos un juez de primera instancia con la voluntad, los recursos y la audacia para embarcarse en una empresa semejante. Ello por no hablar de los serios cuestionamientos que podría generar para este hipotético juez de primera instancia el apartamiento manifiesto de diversas normas procesales (como ha sucedido en "Mendoza"), especialmente en un escenario judicial mayormente conservador como el argentino." (Verbic, 2013:283).
} 
en Seminario en Latinoamérica de Teoría Constitucional y Política.

Bibiloni, Héctor (2005) "Derecho procesal ambiental”, Lexis Nexis, Ciudad Autónoma de Buenos Aires.

Cafferatta, N. (2008) "Sentencia colectiva ambiental en el caso Riachuelo" en Revista de Derecho Ambiental (Agosto).

Chayes, Abram (2017) "El rol del Juez en el litigio de interés público", traducción Milantta, O. y Verbic, F. en Revista de Processo $\mathrm{N}^{\circ} 268$, (Junio).

Giannini, Leandro J. (2009) "Los procesos colectivos en la Ley General Ambiental. Propuestas de reforma" en VVAA (Coord.:Berizonce, Roberto O.), Aportes para una justicia más transparente, La Plata, Platense, pp. 105-169.

Gordillo, A. (2013) "Tratado de Derecho Administrativo", Tomo 2, Capítulo 11: "El procedimiento de audiencia pública" página 450. Consultado en https://www.gordillo.com/pdf_tomo2/ capitulo11.pdf" el 04/02/2019.

Verbic, F. (2015) "La Corte Suprema Argentina y la construcción del derecho constitucional a un debido proceso colectivo" en Int'l Journal of Procedural Law, volumen 5, $\mathrm{N}^{\circ} 1$.

Verbic, F. (2013) "El remedio estructural de la causa "Mendoza". Antecedentes, principales características y algunas cuestiones planteadas durante los primeros tres años de su implementación" en ANALES No 43 - Facultad de Cs. Jurídicas y Sociales. U.N.L.P. 